Also available at http://amc.imfm.si

ISSN 1855-3966 (printed edn.), ISSN 1855-3974 (electronic edn.)

ARS MATHEMATICA CONTEMPORANEA 6 (2013) 279-288

\title{
Unordered multiplicity lists of wide double paths
}

\author{
Aleksandra Erić \\ Faculty of Civil Engineering, University of Belgrade, 11000 Belgrade, Serbia \\ C. M. da Fonseca * \\ Department of Mathematics, University of Coimbra, 3001-501 Coimbra, Portugal
}

Received 20 February 2012, accepted 18 September 2012, published online 19 November 2012

\begin{abstract}
Recently, Kim and Shader analyzed the multiplicities of the eigenvalues of a $\Phi$-binary tree. We carry this discussion forward extending their results to a larger family of trees, namely, the wide double path, a tree consisting of two paths that are joined by another path. Some introductory considerations for dumbbell graphs are mentioned regarding the maximum multiplicity of the eigenvalues. Lastly, three research problems are formulated.
\end{abstract}

Keywords: Graph, tree, matrix, eigenvalues, multiplicities, inverse eigenvalue problem.

Math. Subj. Class.: 15A18, 05C50

\section{Preliminaries}

For a given $n \times n$ real symmetric matrix $A=\left(a_{i j}\right)$, we define the graph of $A$, and write $G(A)$, as the undirected graph whose vertex set is $\{1, \ldots, n\}$ and edge set is $\{i j \mid i \neq$ $j$ and $\left.a_{i j} \neq 0\right\}$. On the other hand, for a given (weighted) graph $G$, we may define $A(G)=\left(a_{i j}\right)$ to be the (real) symmetric matrix whose graph $G(A)$ is $G$. We focus our attention to the set

$$
\mathcal{S}(G)=\left\{A \in \mathbb{R}^{n \times n} \mid A=A^{T} \text { and } G(A)=G\right\},
$$

i.e., the set of all symmetric matrices sharing a common graph $G$ on $n$ vertices. Nevertheless, all results can easily be extended to complex Hermitian matrices.

If $G$ is a tree, then the matrix $A(G)$ is called acyclic. In particular, if $G$ is a path, we order the vertices of $G$ such that $A(G)$ is a tridiagonal matrix.

We will often omit the mention of the graph of the matrix if it is clear from the context.

*This research was supported by CMUC - Centro de Matemática da Universidade de Coimbra and FCT Fundação para a Ciência e a Tecnologia, through European program COMPETE/FEDER.

E-mail addresses: eric@grf.rs (Aleksandra Erić), cmf@mat.uc.pt (C. M. da Fonseca) 
Let us denote the (algebraic) multiplicity of the eigenvalue $\theta$ of a symmetric matrix $A=A(G)$ by $m_{A}(\theta)$. The $(n-1) \times(n-1)$ principal submatrix, formed by the deletion of row and column indexed by $i$, which is equivalent to removing the vertex $i$ from $G$, is designated by $A(G \backslash i)$.

Among the linear algebra community, most of the results on multiplicities of eigenvalues are mainly confined to trees motivated by the Parter-Wiener Theorem [16] and to Cauchy's Interlacing Theorem.

For a more detailed account on the subject the reader is referred to [16]. We remark that the Parter-Wiener Theorem was reformulated in the survey work [7], by the second author, motivated by the earlier seminal work of C. Godsil on matchings polynomials [9, 10, 11]. The same approach produced a result for the multiplicities of an eigenvalue of a matrix involving certain paths of the underlying graph, with many interesting applications to general graphs [4].

Theorem 1.1. [6, 8] Let $P$ be a path that does not contain any edge of any cycle in $G$. Then

$$
m_{A(G \backslash P)}(\theta) \geqslant m_{A(G)}(\theta)-1 .
$$

Since a tree has no cycles, the inequality (1.1) is true for any path in a tree, which generalizes a result for the standard adjacency acyclic matrices [9].

The inequality (1.1) can provide us an upper bound for the multiplicity of an eigenvalue of a graph. The next result was established by R.A. Beezer in [3, Lemma 2.1] and it gives a lower bound. It was originally stated for standard adjacency matrices, but it can be proved for weighted adjacency matrices.

Lemma 1.2. Let us suppose that $H_{1}, \ldots, H_{k}$ be graphs, and let $v_{1}, \ldots, v_{t}$ be additional vertices. Construct a graph $H$ by adding new edges that have one endpoint in the set $\left\{v_{1}, \ldots, v_{t}\right\}$ and the other endpoint in a vertex of some $H_{i}$. If

$$
A=\left(\begin{array}{ccc|c}
A_{1} & & 0 & \\
& \ddots & & C^{T} \\
0 & & A_{k} & \\
\hline & C & & D
\end{array}\right) \in S(H),
$$

where $A_{i} \in \mathcal{S}\left(H_{i}\right)$, for $i=1 \ldots k, D$ is a real diagonal block, and $C$ has $t$ rows, then

$$
m_{A}(\lambda) \geqslant \sum_{i=1}^{k} m_{A_{i}}(\lambda)-t .
$$

We remark that (1.2) is a special case of Cauchy-type interlacing theorems for block Hermitian matrices. In fact, if $\lambda$ is an eigenvalue of the upper block decomposition $A_{1} \oplus$ $\cdots \oplus A_{k}$, a block vector calculation shows that the dimension the eigenspace of $\lambda$ of $A$ is at least as great as the dimension of the intersection of the eigenspace of $\lambda$ of $B$ and the null space of $C$ (for more details the reader is referred to [15]).

Interestingly, Lemma 1.2 provides us an algorithm construct matrices of certain graphs where the maximum multiplicity is attained. For example, let us consider the $(4,3)$-tadpole graph $T$ 


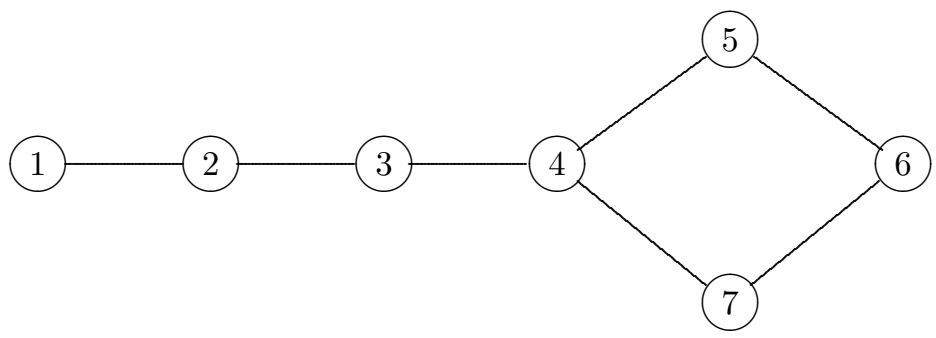

Considering the path joining vertices 1 and 4 in (1.1) we see that, for any eigenvalue $\lambda$ of $A \in \mathcal{S}(T), m_{A}(\lambda) \leqslant 2$ (see also [2, 8]). On the other hand, from (1.2), setting $A_{1}$ for the Jacobi matrix whose graph is the path joining vertices 1 and 2 (an edge) and $A_{2}$ for the matrix whose graph is the cycle containing vertices $4,5,6$, and 7 , we have

$$
m_{A}(\lambda) \geqslant m_{A_{1}}(\lambda)+m_{A_{2}}(\lambda)-1 \geqslant 1+2-1=2 .
$$

Therefore, if we want to construct a matrix in $\mathcal{S}(T)$ with an eigenvalue, say $\sqrt{2}$ of maximal multiplicity, we may set

$$
A_{1}=\left(\begin{array}{rr}
1 & 1 \\
1 & -1
\end{array}\right)
$$

and

$$
A_{2}=\left(\begin{array}{rrrr}
0 & 1 & 0 & -1 \\
1 & 0 & 1 & 0 \\
0 & 1 & 0 & 1 \\
-1 & 0 & 1 & 0
\end{array}\right)
$$

constructed as in [5]. Then any matrix of the form

$$
A=\left(\begin{array}{rrrrrrr}
-1 & 1 & 0 & 0 & 0 & 0 & 0 \\
1 & 1 & * & 0 & 0 & 0 & 0 \\
0 & * & * & * & 0 & 0 & 0 \\
0 & 0 & * & 0 & 1 & 0 & -1 \\
0 & 0 & 0 & 1 & 0 & 1 & 0 \\
0 & 0 & 0 & 0 & 1 & 0 & 1 \\
0 & 0 & 0 & -1 & 0 & 1 & 0
\end{array}\right)
$$

has $\sqrt{2}$ (and, in this case, $-\sqrt{2}$ too) as an eigenvalue of maximal multiplicity 2 .

In general, for a given $(m, n)$-tadpole $G_{m, n}$, if $\lambda$ is an eigenvalue of $A \in \mathcal{S}\left(G_{m, n}\right)$ but not eigenvalue of $A\left(C_{m}\right)$, then $\lambda$ is simple, from (1.1).

In this note, we show how to use inequality (1.1) to generalize a result on the maximum multiplicity of an eigenvalue of a $\Phi$-binary tree due to Kim and Shader [18] to a more general family of trees: a wide double path. A wide double path is consists of two paths that are joined by another path. Unordered multiplicity lists for two classes of wide double paths are established generalizing previous results. At the end three new research problems are pointed out.

First, we show how combining both bounds (1.1) and (1.2) in order to give necessary and sufficient conditions for an eigenvalue has maximal multiplicity in the case of a dumbbell graph. 


\section{Dumbbell graphs}

A dumbbell graph is obtained by joining two cycles by a path. We will assume that the length of this path is greater than 2. Nevertheless, all results are true for lower lengths, with slight modifications.

Dumbbells graphs are a special class of bicyclic graphs, i.e., connected graphs in which the number of edges equals the number of vertices plus one. These graphs are well considered in graph theory, combinatorics, and optimization literature [12, 13, 14, 19, 20, 21$]$. Much attention has recently been paid to the spectral properties of these non-acyclic graphs [22, 23].

We start this section establishing an upper bound for the multiplicity of an eigenvalue of a dumbbell graph.

Proposition 2.1. The maximum multiplicity of an eigenvalue of a dumbbell graph is 3.

Proof. Let $P$ be the path intersecting both pendant cycles of the dumbbell graph $D$. Observe that the (disconnect) subgraph $D \backslash P$ is the union of two paths. Then, from (1.1),

$$
m_{A(D)}(\lambda) \leqslant m_{A(D \backslash P)}(\lambda)+1 \leqslant 2+1=3,
$$

for any eigenvalue $\lambda$ of $D$.

Next we characterize the matrices where an eigenvalue attains the maximum multiplicity.

Proposition 2.2. Let $D$ be a dumbbell graph and let $P$ be the path intersecting both pendant cycles $C_{1}$ and $C_{2}$ at the vertices $v_{1}$ and $v_{\ell}$, respectively. If $\lambda$ is an eigenvalue of $A \in \mathcal{S}(D)$ of multiplicity 3 , then $\lambda$ is an eigenvalue of $A\left(C_{1} \backslash v_{1}\right)$ and of $A\left(C_{2} \backslash v_{\ell}\right)$.

Proof. Again, by Theorem 1.1, if $m_{A(D)}(\lambda)=3$, then

$$
m_{A(D \backslash P)}(\lambda) \geqslant 3-1=2 .
$$

Since $D \backslash P=\left(C_{1} \backslash v_{1}\right) \cup\left(C_{2} \backslash v_{\ell}\right)$ and both $C_{1} \backslash v_{1}$ and $C_{2} \backslash v_{\ell}$ are paths, $\lambda$ must be an eigenvalue of each $A\left(C_{1} \backslash v_{1}\right)$ and of $A\left(C_{2} \backslash v_{\ell}\right)$.

Corollary 2.3. Let $D$ be a dumbbell graph and let $P=v_{1} v_{2} \cdots v_{\ell}$ be the path intersecting both pendant cycles $C_{1}$ and $C_{2}$ at the vertices $v_{1}$ and $v_{\ell}$, respectively. If $\lambda$ is an eigenvalue of $A \in \mathcal{S}(D)$ of multiplicity 3 , then $\lambda$ is an eigenvalue of both $A\left(C_{1}\right)$ and $A\left(C_{2}\right)$.

Proof. Considering the path $P^{\prime}=v_{2} \cdots v_{\ell}$ and (1.1), we have

$$
m_{A\left(D \backslash P^{\prime}\right)}(\lambda) \geqslant 2 \text {. }
$$

Now we only have to observe that $D \backslash P^{\prime}=C_{1} \cup\left(C_{2} \backslash v_{\ell}\right)$ and $m_{A\left(C_{2} \backslash v_{\ell}\right)}(\lambda)=1$.

Note that we can conclude a result more general than Corollary 2.3. In fact, $\lambda$ should be an eigenvalue of any tadpole graph obtaining by joining, for example, the path $v_{i} \cdots v_{\ell}$, for any $i=2, \ldots, \ell-1$, to the cycle $C_{2}$ at $v_{\ell}$.

The next result is a straightforward consequence of (1.1). 
Proposition 2.4. Let $D$ be a dumbbell graph and let $P$ be the path intersecting both pendant cycles $C_{1}$ and $C_{2}$ at the vertices $v_{1}$ and $v_{\ell}$, respectively. If $\lambda$ is an eigenvalue of $A \in \mathcal{S}(D)$ but neither an eigenvalue of $A\left(C_{1} \backslash v_{1}\right)$ nor of $A\left(C_{2} \backslash v_{\ell}\right)$ or neither an eigenvalue of $A\left(C_{1}\right)$ nor of $A\left(C_{2}\right)$, then $m_{A}(\lambda)=1$, i.e., $\lambda$ is a simple eigenvalue of $A$.

As we mentioned before, Lemma 1.2 provides an interesting algorithm producing matrices of certain graphs with eigenvalues of maximum multiplicity. For, let us consider the real number $\lambda$. For a given path of order $k_{1}$, let $A_{1}$ be a tridiagonal matrix of order $k_{1}$, with eigenvalue $\lambda$, using (according Jean Favard) the simple et élégant Wendroff's algorithm [24], appeared a long time ago but somehow has not received so far the appropriate consideration by the linear algebra community. Now, using the general algorithm established in [5], it is possible to construct periodic Jacobi matrices, say $A_{2}$ and $A_{3}$, whose cycles are of orders $k_{2}$ and $k_{3}$, respectively, with $\lambda$ being an eigenvalue of both matrices. Let us set

$$
A=\left(\begin{array}{ccc|cc}
A_{1} & & & & \\
& A_{2} & & x^{T} & y^{T} \\
& A_{3} & & \\
\hline x & & 0 & 0 \\
& y & & 0 & 0
\end{array}\right) \in \mathcal{S}(H),
$$

where $x$ is the 0,1 vector with 1 's in the position 1 and $k_{1}+1$ and 0 elsewhere, and, analogously, $y$ is the 0,1 vector with 1 's in the position $k_{1}$ and $k_{1}+k_{2}+1$ and 0 elsewhere. Then $\lambda$ is an eigenvalue of $A$ of multiplicity 3. In fact, from (1.2),

$$
m_{A}(\lambda) \geqslant m_{A_{1}}(\lambda)+m_{A_{2}}(\lambda)+m_{A_{3}}(\lambda)-2=1+2+2-2=3 .
$$

\section{Maximum multiplicities}

We now turn back our attention to a family of binary trees. Recall that a binary tree is a tree such that the degree of each vertex is no more than three. In this section we will consider the family constituted by the trees of the following form: take five paths $P_{1}, \ldots, P_{5}$ and two vertices $u$ and $v$; join any terminal vertex of $P_{1}, P_{2}$, and $P_{5}$ to $u$; the other terminal vertex of $P_{5}$ and any terminal vertex of $P_{3}$ and of $P_{4}$ are added to $v$. These trees can also be seen as consisting of two paths that are joined by another path. Therefore, we will call them wide double paths. The paths $P_{1}, \ldots, P_{4}$ are the legs (or branches) of such tree.

In [18] Kim and Shader studied several spectral properties of the so-called $\Phi$-binary trees. It is a particular case of the trees under discussion now: $P_{5}$ has size 1 (i.e., a single vertex) and the longest legs among the four legs are connected to different terminal vertices.

Theorem 3.1. Let $T$ be a wide double path and $A \in \mathcal{S}(T)$. Then the maximum multiplicity of an eigenvalue of $A$ is 3 .

Proof. We only have to apply (1.1), for example, to the path $P_{1}-u-P_{5}-v-P_{3}$.

Theorem 3.2. For a given wide double path $T, \lambda$ is an eigenvalue of $A \in \mathcal{S}(T)$ of maximum multiplicity if and only if $\lambda$ is an eigenvalue of each path $P_{1}, \ldots, P_{5}$.

Proof. Set $A_{i}=A\left(P_{i}\right)$, for $i=1, \ldots, 5$. Let us assume first that $m_{A}(\lambda)=3$. Considering the path $P_{1}-u-P_{5}-v-P_{3}$ in $T$, from (1.1), it follows

$$
m_{A_{2}}(\lambda)+m_{A_{4}}(\lambda) \geqslant 3-1=2 .
$$


Thus, $m_{A_{2}}(\lambda)=m_{A_{4}}(\lambda)=1$. Analogously, we prove $m_{A_{1}}(\lambda)=m_{A_{3}}(\lambda)=1$. It remains to prove that $m_{A_{5}}(\lambda)=1$. In fact, since $P_{5}$ can be obtained from $T$ deleting the paths $P_{1}-u-P_{2}$ and $P_{3}-u-P_{4}$, we have again, from (1.1),

$$
1 \geqslant m_{A_{5}}(\lambda) \geqslant m_{\tilde{A}}(\lambda)-1 \geqslant m_{A}(\lambda)-2=1,
$$

where $\tilde{A}=A(H)$, with $H$ being the generalized star with center $u$ and legs $P_{1}, P_{2}$, and $P_{5}$.

Conversely, if $m_{A_{i}}(\lambda)=1$, for $i=1, \ldots, 5$, then

$$
3 \geqslant m_{A}(\lambda) \geqslant \sum_{i=1}^{5} m_{A_{i}}(\lambda)-2=3 .
$$

from Theorem 3.1 and (1.2).

Let us set $\ell_{i}$ for the order of the path $P_{i}$, with $i=1, \ldots, 5$, in a wide double path $W$.

Corollary 3.3. The number $n_{3}$ of eigenvalues with multiplicity 3 of a wide double path is at most $\min \left\{\ell_{1}, \ldots, \ell_{5}\right\}$.

Corollary 3.4. [18, Theorem 2(a)] Let $T$ be a $\Phi$-binary tree and $A \in \mathcal{S}(T)$. Then there are no eigenvalues of multiplicity 4 or more, and the number $n_{3}$ of eigenvalues with multiplicity 3 is at most one. Furthermore, if $\lambda \in \sigma(A)$ with $m_{A}(\lambda)=3$, then the diagonal entry of $A$ corresponding to the axis vertex of $T$ is $\lambda$.

We now investigate the eigenvalues of multiplicity 2 . The first result is a consequence of Lemma 1.2 and Theorem 3.2.

Lemma 3.5. Let $T$ be a wide double path and let $A \in \mathcal{S}(T)$. If $\lambda \in \sigma(A)$ is an eigenvalue of exactly four of the paths $P_{1}, \ldots, P_{5}$, then $m_{A}(\lambda)=2$.

As before, $n_{2}$ denotes the number of eigenvalues of multiplicity 2 of a given matrix.

Theorem 3.6. Let $W$ be a wide double path and $r=\min \left\{\ell_{i}+\ell_{j} \mid i=1,2, j=3,4\right\}$. Then

$$
n_{2} \leqslant r-2 n_{3}
$$

Proof. By Theorem 3.2, if $\lambda_{1}, \ldots, \lambda_{n_{3}}$ are the distinct eigenvalues of $A$ of multiplicity 3 , then they must be (simple) eigenvalues of both $A\left(P_{2}\right)$ and $A\left(P_{4}\right)$. Taking into account Lemma 3.5, the inequality (3.1) follows.

We remark that Theorem 3.2 is in fact much more general. An analogous result can be proved for any generalized caterpillar, i.e., a tree for which removing the legs produces a path, and the maximum multiplicity of any eigenvalue is equal to the number of legs minus one. In particular we have the following result.

Lemma 3.7. Let $S$ be a generalized star and $A \in \mathcal{S}(S)$. Then $m_{A}(\lambda)=2$ if and only if $\lambda$ is an eigenvalue of each leg.

Theorem 3.8. Let $T$ be a wide double path and $A \in \mathcal{S}(T)$. Then $m_{A}(\lambda)=2$ if and only if $\lambda$ is a simple eigenvalue of the paths $P_{1}, P_{2}$, and of the generalized star with center $v$ and legs $P_{3}, P_{4}, P_{5}$, or is a simple eigenvalue of the paths $P_{3}, P_{4}$, and of the generalized star with center $u$ and legs $P_{1}, P_{2}, P_{5}$. 
Proof. Let us assume that $m_{A}(\lambda)=2$. From (1.1), for any path $P$ in $T, m_{A(T \backslash P)}(\lambda) \geqslant 1$. Therefore, if $\lambda$ is not an eigenvalue of $P_{1}\left(P_{2}\right)$, then it must be an eigenvalue of both $P_{3}$ and $P_{4}$. Moreover, if $S$ denotes the generalized star with center $u$ and legs $P_{1}, P_{2}, P_{5}$, then $m_{A(S)}(\lambda) \geqslant 1$. The other assertion is set in a similar fashion.

The converse follows from (1.1) and (1.2).

We now address the question on the number $n_{1}$ of simple eigenvalues of $A \in \mathcal{S}(W)$. Since

$$
n=n_{1}+2 n_{2}+3 n_{3}=\ell_{1}+\cdots+\ell_{5}+2,
$$

on the one hand, we have

$$
n_{1} \leqslant n
$$

In fact, the equality is attained when we construct $A\left(L_{1}\right), \ldots, A\left(L_{5}\right)$, with distinct eigenvalues. On the other hand,

$$
\begin{aligned}
n_{1} & =\ell_{1}+\ell_{2}+\ell_{3}+\ell_{4}+\ell_{5}+2-2 n_{2}-3 n_{3} \\
& \geqslant \ell_{1}+\ell_{2}+\ell_{3}+\ell_{4}+\ell_{5}+2-2 r+n_{3} \\
& \geqslant\left|\ell_{1}-\ell_{2}\right|+\left|\ell_{3}-\ell_{4}\right|+\ell_{5}+2 .
\end{aligned}
$$

Interestingly, we observe that, from the lower bound (3.3), any matrix in $\mathcal{S}(W)$ must have at least $\ell_{5}+2$ simple eigenvalues. This generalizes [18, Corollary 3 ].

\section{Unordered multiplicity lists}

Recall that if $m_{1} \geqslant \cdots \geqslant m_{k}$, with $m_{1}+\cdots+m_{k}=n$, are the multiplicities of the distinct eigenvalues of an $n$-by- $n$ symmetric matrix $A$, then $\left(m_{1}, \ldots, m_{k}\right)$ is the unordered multiplicity list (or list) of the eigenvalues of $A$. By unordered multiplicity list of a graph $G$ we mean the set of unordered multiplicity lists for all matrices in $\mathcal{S}(G)$.

Without loss of generality, we will assume that $\ell_{1} \geqslant \ell_{2}$ and $\ell_{3} \geqslant \ell_{4}$. Moreover, we convention that for a finite sequence of real numbers $a_{1}, \ldots, a_{i}$, with $i \leqslant 0$, is empty.

We are able now to find the unordered multiplicity lists of the wide double path under discussion.

Theorem 4.1. Let $W$ be a wide double path of order $n$, with $\ell_{1} \geqslant \ell_{2}$ and $\ell_{3} \geqslant \ell_{4}$. Then the set of unordered multiplicity lists of $W$ consists of the positive integer lists of the form

$$
(\underbrace{3, \ldots, 3}_{n_{3}}, \underbrace{2, \ldots, 2}_{n_{2}}, \underbrace{1, \ldots, 1}_{n_{1}}) \text {, }
$$

with $0 \leqslant n_{3} \leqslant \min \left\{\ell_{2}, \ell_{4}, \ell_{5}\right\}, 0 \leqslant n_{2} \leqslant \ell_{2}+\ell_{4}-2 n_{3}$, and $n_{1}=n-2 n_{2}-3 n_{3}$.

Proof. From our discussion in the previous section, it is clear that any unordered multiplicity list of $W$ is of the form (4.1).

Conversely, let $S_{1}$ (resp., $S_{2}$ ) be the generalized star with center vertex $u$ (resp., $v$ ) and legs $L_{1}, L_{2}, L_{5}$ (resp., $\left.L_{3}, L_{4}, L_{5}\right)$. Now, for $k=0, \ldots, \min \left\{\ell_{2}, \ell_{4}, \ell_{5}\right\}, p=0, \ldots, \ell_{2}-k$, and $q=0, \ldots, \ell_{4}-k$, let us consider the $\ell_{1}+\ell_{5}-k+p+1$ distinct real numbers

$$
\beta_{1}, \ldots, \beta_{\ell_{4}-k-q}, \theta_{1}, \ldots, \theta_{\ell_{1}-\ell_{4}+\ell_{5}+p+q+1}
$$


strictly interlacing with the $\ell_{1}+\ell_{5}-k+p$ (distinct) real numbers

$$
\lambda_{1}, \ldots, \lambda_{\ell_{5}}, \alpha_{1}, \ldots, \alpha_{\ell_{1}-k}, \tilde{\alpha}_{\ell_{2}-k-p+1}, \ldots, \tilde{\alpha}_{\ell_{2}-k},
$$

and the $\ell_{3}+\ell_{5}-k+q+1$ distinct real numbers

$$
\alpha_{1}, \ldots, \alpha_{\ell_{2}-k-p}, \mu_{1}, \ldots, \mu_{\ell_{3}-\ell_{2}+\ell_{5}+q+p+1}
$$

strictly interlacing with the $\ell_{3}+\ell_{5}-k+q$ (distinct) real numbers

$$
\lambda_{1}, \ldots, \lambda_{\ell_{5}}, \beta_{1}, \ldots, \beta_{\ell_{3}-k}, \tilde{\beta}_{\ell_{4}-k-q+1}, \ldots, \tilde{\beta}_{\ell_{4}-k} .
$$

Now we consider $A \in \mathcal{S}(W)$ such that

$$
\begin{aligned}
& \sigma\left(A\left(L_{5}\right)\right)=\left\{\lambda_{1}, \ldots, \lambda_{k}, \lambda_{k+1}, \ldots, \lambda_{\ell_{5}}\right\} \\
& \sigma\left(A\left(L_{1}\right)\right)=\left\{\lambda_{1}, \ldots, \lambda_{k}, \alpha_{1}, \ldots, \alpha_{\ell_{2}-k-p}, \alpha_{\ell_{2}-k-p+1}, \ldots, \alpha_{\ell_{1}-k}\right\} \\
& \sigma\left(A\left(L_{2}\right)\right)=\left\{\lambda_{1}, \ldots, \lambda_{k}, \alpha_{1}, \ldots, \alpha_{\ell_{2}-k-p}, \tilde{\alpha}_{\ell_{2}-k-p+1}, \ldots, \tilde{\alpha}_{\ell_{2}-k}\right\} \\
& \sigma\left(A\left(S_{1}\right)\right)=\left\{\lambda_{1}, \lambda_{1}, \ldots, \lambda_{k}, \lambda_{k}, \alpha_{1}, \ldots, \alpha_{\ell_{2}-k-p}, \beta_{1}, \ldots, \beta_{\ell_{4}-k-q}, \theta_{1}, \ldots,\right. \\
&\left.\theta_{\ell_{1}-\ell_{4}+\ell_{5}+p+q+1}\right\} \\
& \sigma\left(A\left(L_{3}\right)\right)=\left\{\lambda_{1}, \ldots, \lambda_{k}, \beta_{1}, \ldots, \beta_{\ell_{4}-k-q}, \beta_{\ell_{4}-k-q+1}, \ldots, \beta_{\ell_{3}-k}\right\} \\
& \sigma\left(A\left(L_{4}\right)\right)=\left\{\lambda_{1}, \ldots, \lambda_{k}, \beta_{1}, \ldots, \beta_{\ell_{4}-k-q}, \tilde{\beta}_{\ell_{4}-k-q+1}, \ldots, \tilde{\beta}_{\ell_{4}-k}\right\} \\
& \sigma\left(A\left(S_{2}\right)\right)=\left\{\lambda_{1}, \lambda_{1}, \ldots, \lambda_{k}, \lambda_{k}, \beta_{1}, \ldots, \beta_{\ell_{4}-k-q}, \alpha_{1}, \ldots, \alpha_{\ell_{2}-k-p}, \mu_{1}, \ldots,\right. \\
&\left.\mu_{\ell_{3}-\ell_{2}+\ell_{5}+q+p+1}\right\},
\end{aligned}
$$

The existence of the Jacobi matrices is granted by [24] and the two generalized stars by [17, Theorem 11].

It is clear that the unordered multiplicity list of $A$ is

$$
(\underbrace{3, \ldots, 3}_{t_{3}}, \underbrace{2, \ldots, 2}_{t_{2}}, \underbrace{1, \ldots, 1}_{t_{1}}),
$$

with $t_{3}=k, t_{2}=\ell_{2}+\ell_{4}-2 k-p-q$, and $t_{1}=\left(\ell_{1}-\ell_{2}\right)+\left(\ell_{3}-\ell_{4}\right)+\ell_{5}+2+k+2(p+q)$. Note that $0 \leqslant k+2(p+q) \leqslant 2\left(\ell_{2}+\ell_{4}\right)$.

Observe that with $1=\ell_{5} \leqslant \ell_{2}, \ell_{4}, \leqslant \ell_{1}, \ell_{3}$, we are able to recover the results in [18] for $\Phi$-binary trees. Moreover, Theorem 4.1 can also be applied for $\ell_{5}=0[1,17]$.

Finally, a tree is minimal provided there is a matrix such that number of distinct eigenvalues is equal to the diameter (counting edges) plus one. From Theorem 4.1, we conclude that the wide double paths are minimal, for $\ell_{2}, \ell_{4} \leqslant \ell_{1}, \ell_{3} \leqslant \ell_{5}$. In fact, with $t_{3}=0$, $t_{2}=\ell_{2}+\ell_{4}$, and $t_{1}=\left(\ell_{1}-\ell_{2}\right)+\left(\ell_{3}-\ell_{4}\right)+\ell_{5}+2$, since the number of distinct eigenvalues is $\ell_{1}+\ell_{3}+\ell_{5}+2$ and the diameter is $\ell_{1}+\ell_{3}+\ell_{5}+1$.

\section{Open problems}

In this paper, we provided the solution for the inverse eigenvalue problem of a wide double path, generalizing the results for the very particular case of the family of the so-called $\Phi$ binary trees, recently established. The approach adopted here is different, offering a general result with a more concise proof.

A natural generalization a wide double path is when we have more than 2 legs adjacent to the "central" vertices $u$ and $v$. Let us suitably call such trees as wide double generalized stars. 
Problem 1. Characterize the unordered multiplicity lists of a wide double generalized star.

It seems this is not a difficult problem to handle and an elegant characterization similar to Theorem 4.1 may be achieved.

A more hard problem is related an analogous characterization for binary trees.

Problem 2. Characterize the unordered multiplicity lists of a binary tree.

Our results may also be seen as the starting point for a more meaningful study:

Problem 3. What are the unordered multiplicity lists of trees having maximum multiplicity 3 ?

Of course, from our approach, the previous question can be extended to general graphs. Probably new techniques will need to be developed for this attractive and vast area of research. Some computational experiments allow us to assert that there will be some surprising multiplicity lists.

\section{References}

[1] F. Barioli and S.M. Fallat, On the eigenvalues of generalized and double generalized stars, Linear Multilinear Algebra 53 (2005), 269-291.

[2] F. Barioli, S.M. Fallat and L. Hogben, Computation of minimal rank and path cover number for certain graphs, Linear Algebra Appl. 392 (2004), 289-303.

[3] R.A. Beezer, Trees with very few eigenvalues, J. Graph Theory 14 (1990), 509-517.

[4] A. Erić and C.M. da Fonseca, Some consequences of an inequality on the spectral multiplicity of graphs, submitted.

[5] R. Fernandes and C.M. da Fonseca, The inverse eigenvalue problem for Hermitian matrices whose graphs are cycles, Linear Multilinear Algebra 57 (2009), 673-682.

[6] C.M. da Fonseca, A note on the multiplicities of the eigenvalues of a graph, Linear Multilinear Algebra 53 (2005), 303-307.

[7] C.M. da Fonseca, On the multiplicities of eigenvalues of a Hermitian matrix whose graph is a tree, Ann. Mat. Pura Appl. 187 (2008), 251-261.

[8] C.M. da Fonseca, A lower bound for the number of distinct eigenvalues of some symmetric matrices, Electron. J. Linear Algebra 21 (2010), 3-11.

[9] C.D. Godsil, Spectra of trees, Ann. Discrete Math. 20 (1984), 151-159.

[10] C.D. Godsil, Algebraic Combinatorics, Chapman and Hall, New York and London, 1993.

[11] C.D. Godsil, Algebraic matching theory, Electron. J. Combin. 2 (1995), \#R8.

[12] J.L. Gross and J. Chen, Algebraic specification of interconnection network relationships by permutation voltage graph mappings, Math. Systems Theory 29 (1996), 451-470.

[13] P. Hansen, A. Hertz, R. Kilani, O. Marcotte and D. Schindl, Average distance and maximum induced forest, J. Graph Theory $\mathbf{6 0}$ (2009), 31-54.

[14] P. Hell and X. Zhu, Adaptable chromatic number of graphs, European J. Combin. 29 (2008), 912-921.

[15] R.A. Horn, N.H. Rhee and W. So, Eigenvalue inequalities and equalities, Linear Algebra Appl. 270 (1998), 29-44. 
[16] C.R. Johnson, A. Leal Duarte and C.M. Saiago, The Parter-Wiener theorem: refinement and generalization, SIAM J. Matrix Anal. Appl. 25 (2003), 352-361.

[17] C.R. Johnson, A. Leal Duarte and C.M. Saiago, Inverse eigenvalue problems and lists of multiplicities of eigenvalues for matrices whose graph is a tree: The case of generalized stars and double generalized stars, Linear Algebra Appl. 373 (2003), 311-330.

[18] I.-J. Kim and B.L. Shader, Unordered multiplicity lists of a class of binary trees, Linear Algebra Appl. (2011), doi:10.1016/j.1aa.2011.07.006

[19] S. Kratsch and P. Schweitzer, Isomorphism for graphs of bounded feedback vertex set number, in: H. Kaplan (ed.), Algorithm Theory - SWAT 2010, Lecture Notes in Computer Science 6139, Springer, 2010, 81-92.

[20] J.H. Kwak and J. Lee, Isomorphism classes of cycle permutation graphs, Discrete Math. 105 (1992), 131-142.

[21] S.-M. Lee, K.-J. Chen and Y.-C. Wang, On the edge-graceful spectra of cycles with one chord and dumbbell graphs, Congr. Numer. 170 (2004), 171-183.

[22] J. Wang, F. Belardo, Q. Huang and E.M. Li Marzi, Spectral characterizations of dumbbell graphs, Electron. J. Combin. 17 (2010), \#R42.

[23] J. Wang, Q. Huang, F. Belardo and E.M. Li Marzi, A note on the spectral characterization of dumbbell graphs, Linear Algebra Appl. 431 (2009), 1707-1714.

[24] B. Wendroff, On orthogonal polynomials, Proc. Amer. Math. Soc. 12 (1961), 554-555. 